\title{
Subject ID
}

National Cancer Institute

\section{Source}

National Cancer Institute. Subject ID. NCI Thesaurus. Code C69258.

An identifier for a person who is the subject in a study. 